\title{
An Efficient Nonconvex Regularization Method for Wavelet Frame Based Compressed Sensing Recovery
}

\author{
Xiao-Juan Yang ${ }^{1}$ and Jin Jing ${ }^{2}$
}

\begin{abstract}
In this paper, we propose a variation model which takes advantage of the wavelet tight frame and nonconvex shrinkage penalties for compressed sensing recovery. We address the proposed optimization problem by introducing a adjustable parameter and a firm thresholding operations. Numerical experiment results show that the proposed method outperforms some existing methods in terms of the convergence speed and reconstruction errors.
\end{abstract}

JEL classification numbers: $68 \mathrm{U} 10,65 \mathrm{~K} 10,90 \mathrm{C} 25,62 \mathrm{H} 35$.

Keywords: Compressed Sensing, Nonconvex, Firm thresholding, Wavelet tight frame.

\footnotetext{
${ }^{1}$ School of Mathematical Sciences, Nanjing Normal University, Taizhou College Taizhou, 225300, P. R. China.

${ }^{2}$ School of Mathematical Sciences, Nanjing Normal University, Taizhou College Taizhou, 225300, P. R. China.
}

Article Info: Received: June 27, 2021. Revised: July 15, 2021.

Published online: July 20, 2021. 


\section{Introduction}

The magnetic resonance imaging (MRI) is widely used in medical diagnoses because of its high resolution and noninvasiveness, and an imaging speed is important in many MRI applications. In recent years, Compressed Sensing (CS) has shown great abilities to accelerate MRI if an image can be sparsely represented.

CS seeks to represent a signal from a small number of linear measurements. We let the vector $x \in \mathbb{R}^{n}$ represents the MR image rearranged to a column vector. The linear measurements are the result of the short and fat measurement matrix $A \in$ $\mathbb{R}^{m \times n}$, with $m \ll n$. In general, $A$ can be represented as the product of a discrete Fourier transform $F \in \mathbb{R}^{n \times n}$ and a undersampling matrix $U \in \mathbb{R}^{m \times n}$, i.e., $A=U F$. One is given the measurements

$$
y=U F x+e
$$

and wants to recover and $x$. Where $e \in \mathbb{R}^{m}$ represents the additive noise, and the noise $e$ is random and only its statistical properties may be known. $m \ll n$ implies that (1) is an underdetermined linear system in $x$. It is well known that the problem of estimating $x$ from $y$ is ill-posed, so additional assumptions must be made about $x$, thus this inverse problem can be solved by adopting some sort of regularization. Images usually have sparse representations (or sparse approximations) in some transformed domains. We assume that the image $x$ is sparse under the representation of some tight frame. In many cases, the sparse solution can be approximated by solving an $l_{1}$ norm regularized minimization problem. Since the tight frame representation is not unique, it is common to divide these $l_{1}$ regularized minimization problems into two subcategories for MR image reconstruction (1), namely the analysis model [1-3] and synthesis model [1,4,5]:

$$
\begin{aligned}
& \text { Analysis : } \min _{x} \lambda\|\Psi x\|_{1}+\frac{1}{2}\|y-U F x\|_{2}^{2} ; \\
& \text { Synthesis : } \min _{\alpha} \lambda\|\alpha\|_{1}+\frac{1}{2}\|y-U F \Phi \alpha\|_{2}^{2},
\end{aligned}
$$

where $\Psi$ is an analysis operator to sparsify the image, $\Phi$ is a synthesis dictionary, $\alpha$ is the corresponding coefficient, and $\lambda$ is the regularization parameter to balance the fidelity term and the regularization term.

Elad et al. studied these two models and offered a geometric explanation of the relation between them [6]. Although the synthesis model has attracted more attention than the analysis model in the past, recent studies show that the latter has its own advantage over the former both theoretically [7,8] and empirically [9]. 
Y.S. Liu et al. in [10] proposed a fast algorithm, called projected iterative softthresholding algorithm (pISTA) which includes two stages, to solved the analysis model (2). In the first stage, the analysis model (2) is rewritten into an equivalent synthesis-like one. In the second stage, the non-smooth terms in the objective function is calculated by the proximal map. By incorporating the acceleration strategy in [11], the authors get the acceleration algorithm pFISTA.

Convex functions are attractive because they can be more reliably minimized than non-convex functions. However, non-convex penalty functions can lead to enhanced sparsity of sparse signals. Nikolova in [12-14] discussed the mathematical properties of the solutions to non-convex regularized signal restoration problems. In [15], Yin et al. proposed a wavelet-TV denoising approach, which used nonconvex penalty function to strongly induce wavelet sparsity. In [16], nonconvex penalty functions have been shown both theoretically and empirically to give better results for compressed sensing than the $l_{1}$ norm, and the authors specifically examined two shrinkages and associated nonconvex penalties - $p$-shrinkage and firm thresholding.

In this paper, in order to induce wavelet-domain sparsity, we propose a nonconvex regularization method for compressed sensing recovery, where we apply a nonconvex penalty due to its strong sparsity-inducing properties.

This paper is outlined as follows. In the next section, in order to combine the advantages of the wavelet tight frame based method and the nonconvex penalty function, we propose a nonconvex regularization model for the problem (1). In this paper, we use firm thresholding, a continuous piecewise-linear approximation of hard thresholding, to compute the minimization involving the non-convex penalty. In section 3, we generalize the idea of the algorithm pISTA in [10] to solve the proposed model. Some numerical experiments are given in section 4 to illustrate the performance of the proposed algorithm.

\section{The proposed model}

Before introducing the new model, we give some necessary notations and definitions. $W=\left[W_{0}^{T}, W_{1,1}^{T}, \cdots, W_{1, J}^{T}, \cdots, W_{Q, 1}^{T}, \cdots, W_{Q, J}^{T}\right]^{T} \in \mathbb{R}^{(J Q+1) n^{2} \times n^{2}}$ be a multi-level wavelet tight frame transform operator (i.e $W^{T} W=I$ ) that converts an image to its wavelet coefficients, in which $Q$ indicates the level of wavelet decomposition and $J$ is the number of high-pass filters that the wavelet system used. So $W f$ is a multi-level wavelet tight frame transform of $f$.

In this paper, the estimated solution for the problem (1) is obtained by minimizing the objective function

$$
\Theta(x)=\lambda \Phi(W x)+\frac{1}{2}\|y-U F x\|_{2}^{2}
$$

where $\Phi(W x)=\sum_{i=1}^{(J Q+1) n^{2}} \phi\left((W x)_{i}\right)$ with 


$$
\phi(x)= \begin{cases}|t|-\frac{t^{2}}{2 \mu}, & \text { if }|t|<\mu, \\ \frac{\mu}{2}, & \text { if }|t| \geq \mu,\end{cases}
$$

and the term $\Phi(W x)$ promotes the sparsity. In the function $\phi, \mu>0$ is a firm thresholding parameter.

Compressed sensing methods rely on the notion of sparsity, which is primarily approximated via the $l_{1}$ norm $[17,18]$. The nature and limitations of this relaxation have been well-studied in [19-22]. Recent work has shown that nonconvex penalty functions have both theoretically and empirically to give better results for compressed sensing than the $l_{1}$ norm [23-25]. In Figure 1, we can see the firm thresholding inducing penalty function can promote sparsity better.

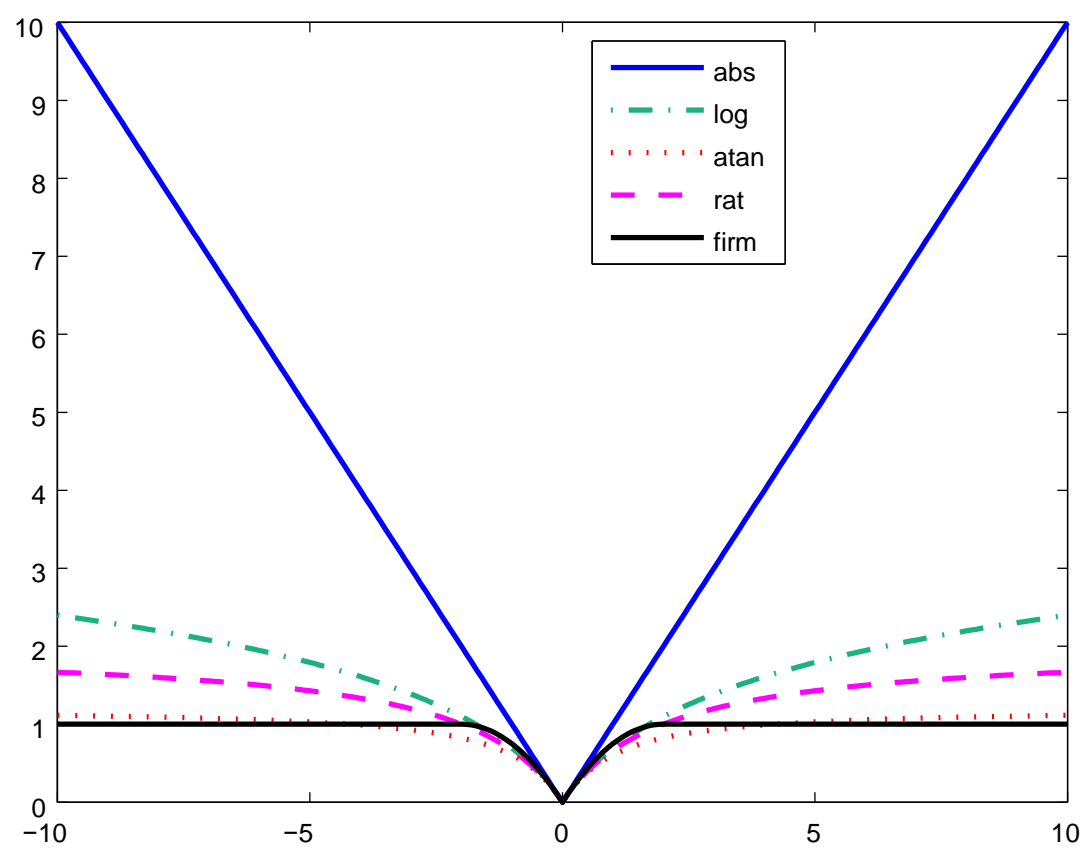

Figure 1: Non-convex penalty

In the model (4), we use the non-convex penalty function $\phi$ to promote sparsity, but we seek to ensure the strict convexity of the objective function $\Theta$.

To find a condition on $\phi$ ensuring $\Theta$ is strictly convex, we rewrite $\Theta$ as follows: 


$$
\Theta(x)=\lambda\|\wedge W x\|_{1}-\frac{\lambda}{2 \mu}\|\wedge W x\|_{2}^{2}+\frac{\lambda \mu}{2}\|(I-\wedge) e\|_{1}+\frac{1}{2}\|y-U F x\|_{2}^{2},
$$

where $\wedge=\operatorname{diag}\left(\wedge_{1}, \cdots, \wedge_{n^{2}}\right)$ is a diagonal matrix with

$$
\wedge_{i}= \begin{cases}1, & \text { if }\left|(W x)_{i}\right|<\mu, \\ 0, & \text { if }\left|(W x)_{i}\right| \geq \mu,\end{cases}
$$

and $e=(1, \cdots, 1)^{T}$ is a vector.

We further denote that

$$
\begin{gathered}
\Theta_{1}(x)=\frac{1}{2}\|y-U F x\|_{2}^{2}-\frac{\lambda}{2 \mu}\|W x\|_{2}^{2} \\
\Theta_{2}(x)=\lambda\|\wedge W x\|_{1}+\frac{\lambda}{2 \mu}\|(I-\wedge) W x\|_{2}^{2}+\frac{\lambda \mu}{2}\|(I-\wedge) e\|_{1}
\end{gathered}
$$

Then

$$
\Theta(x)=\Theta_{1}(x)+\Theta_{2}(x) .
$$

Note that if both $\Theta_{1}(x)$ and $\Theta_{2}(x)$ are strictly convex, then $\Theta(x)$ is strictly convex. We can see if $(U F)^{T}(U F)-\frac{\lambda}{\mu} I$ is positive definite, the function $\Theta_{1}(x)$ is strictly convex. In the following, we discuss the convexity of the function $\Theta_{2}(x)$. Define the function $\varphi(t)$ as the following form

$$
\varphi(t)=\phi(x)+\frac{t^{2}}{2 \mu}= \begin{cases}|t|, & \text { if }|t|<\mu, \\ \frac{\mu}{2}+\frac{t^{2}}{2 \mu}, & \text { if }|t| \geq \mu .\end{cases}
$$

By the definition of $\varphi$, we have that it is twice differentiable on $\mathbb{R} /\{-\mu, 0, \mu\}$. And we have $\varphi_{-}^{\prime}(-\mu)=\varphi_{+}^{\prime}(-\mu), \varphi_{-}^{\prime}(0)<\varphi_{+}^{\prime}(0)$ and $\varphi_{-}^{\prime}(\mu)=\varphi_{+}^{\prime}(\mu)$ by some simple calculating. By Theorem 6.4 of [26], $\varphi$ is strictly convex if it has a strictly increasing right-derivative.

The function $\Theta_{2}(x)$ can be rewritten as a sum of the form

$$
\Theta(x)=\lambda \sum_{i=1}^{(J Q+1) n^{2}} \varphi\left((W x)_{i}\right)
$$


With

$$
\varphi\left((W x)_{i}\right)= \begin{cases}\left|(W x)_{i}\right|, & \text { if }\left|(W x)_{i}\right|<\mu, \\ \frac{\mu}{2}+\frac{(W x)_{i}{ }^{2}}{2 \mu}, & \text { if }\left|(W x)_{i}\right| \geq \mu .\end{cases}
$$

Since the linear transformation can keep the convexity of the function $\varphi(\cdot)$, we get that the function $\Theta_{2}(x)$ is convex. For the convexity of the function $\Theta(x)$, we have

Theorem 2.1 If $(U F)^{T}(U F)-\frac{\lambda}{\mu} I$ is positive definite, then the function $\Theta(x)$ in (4) is strictly convex.

\section{Numerical algorithm}

In this paper, we extend the algorithm pISTA in [10] to solve the proposed model (4). By introducing new auxiliary variables $u=W x$, and note that $W^{T} W=I$, the model (4) can be reformulated as the following constrained optimization problem

$$
\min _{u} \lambda \Phi(\mathrm{u})+\frac{1}{2}\left\|y-U F W^{T} u\right\|_{2}^{2}, \quad \text { s.t. } \quad W x=u .
$$

(7) is equivalent to the following constrained optimization form:

$$
\min _{u \in \text { Range }(W)} \lambda \Phi(\mathrm{u})+\frac{1}{2}\left\|y-U F W^{T} u\right\|_{2}^{2}
$$

The details about the proof of the equivalence can be seen in [10].

As in [10], we introduce an indicator function with

$$
d(u)= \begin{cases}0, & \text { if } u \in \operatorname{Range}(W), \\ \infty, & \text { if } u \notin \operatorname{Range}(W),\end{cases}
$$

then the synthesis-like analysis model (8) can be rewritten as the following unconstrained form

$$
\min _{u} \lambda \Phi(u)+\frac{1}{2}\left\|y-U F W^{T} u\right\|_{2}^{2}+d(u)
$$

In (9), we denote that 


$$
\begin{gathered}
g(u)=\lambda \Phi(u)+d(u), \\
f(u)=\frac{1}{2}\left\|y-U F W^{T} u\right\|_{2}^{2},
\end{gathered}
$$

where $g(u)$ is a non-smooth non-convex function, and $f(u)$ is a smooth function with a $L_{f}$-Lipschitz continuous gradient $\nabla f=\left(W F^{T} U^{T}\right)\left(U F W^{T} u-y\right)$.

We use the ISTA (the iterative soft-thresholding algorithm) in [27] to solve the problem (9)

$$
\begin{aligned}
& u_{k+1}=\operatorname{prox}_{\gamma g}\left(u_{k}-\gamma \nabla f\left(u_{k}\right)\right) \\
& =\arg \min _{u} \lambda \gamma \Phi(u)+\frac{1}{2}\left\|u-\left(u_{k}-\gamma \nabla f\left(u_{k}\right)\right)\right\|_{2}^{2}+d(u) \\
& =\arg \min _{u \in \operatorname{Range}(W)} \lambda \gamma \Phi(u)+\frac{1}{2}\left\|u-\left(u_{k}-\gamma \nabla f\left(u_{k}\right)\right)\right\|_{2}^{2},
\end{aligned}
$$

where $\operatorname{prox}_{f}(x)=\operatorname{argmin}_{z} f(z)+\frac{1}{2}\|z-x\|_{2}^{2}$ is a proximal map, and $\gamma$ is the step size.

Observing that without the constraint $u \in \operatorname{Range}(W),(10)$ can be written as

$$
\begin{aligned}
u_{k+1} & =\arg \min _{u} \lambda \gamma \Phi(u)+\frac{1}{2}\left\|u-\left(u_{k}-\gamma \nabla f\left(u_{k}\right)\right)\right\|_{2}^{2} \\
& =\sum_{i=1}^{(J Q+1) n^{2}} \lambda \gamma \phi\left(u_{i}\right)+\frac{1}{2}\left\|u-\left(u_{k}-\gamma \nabla f\left(u_{k}\right)\right)\right\|_{2}^{2},
\end{aligned}
$$

where $\phi$ is the function defined in (5).

Note that the minimization in (11) is separable. With respect to each component, it is easy to show that the solution of (11) is

$$
\tilde{u}_{k+1}=S_{f i r m}\left(\left(u_{k}-\gamma \nabla f\left(u_{k}\right)\right), \lambda \gamma, \mu\right)
$$

where

$$
S_{\text {firm }}(x, \delta, \mu)=\left\{\begin{array}{cc}
0, & \text { if } \quad|x|<\delta \\
\frac{\mu}{\mu-\delta}(x-\delta \operatorname{sign}(x)), & \text { if } \delta \leq|x| \leq \mu . \\
x, & \text { if } \quad|x|>\mu
\end{array}\right.
$$

Then the solution (10) can be represented as

$$
\begin{aligned}
& \tilde{u}_{k+1}=S_{\text {firm }}\left(u_{k}-\gamma \nabla f\left(u_{k}\right), \lambda \gamma, \mu\right), \\
& u_{k+1}=P_{\text {Range }(W)}\left(\tilde{u}_{k+1}\right),
\end{aligned}
$$

where $P_{\text {Range }(W)}$ is the orthogonal projection operator onto $u \in \operatorname{Range}(W)$. With $\nabla f=\left(W F^{T} U^{T}\right)\left(U F W^{T} u-y\right),(12)$ can be written as 


$$
\begin{aligned}
& \tilde{u}_{k+1}=S_{f i r m}\left(u_{k}+\gamma W F^{T} U^{T}\left(y-U F W^{T} u_{k}\right), \lambda \gamma, \mu\right), \\
& u_{k+1}=W W^{T}\left(\tilde{u}_{k+1}\right),
\end{aligned}
$$

Substituting the second equation of (13) in the first one, we obtain

$$
\begin{aligned}
\tilde{u}_{k+1} & =S_{\text {firm }}\left(u_{k}+\gamma W F^{T} U^{T}\left(y-U F W^{T} u_{k}\right), \lambda \gamma, \mu\right) \\
& =S_{\text {firm }}\left(W W^{T} \tilde{u}_{k}+\gamma W F^{T} U^{T}\left(y-U F W^{T} W W^{T} \tilde{u}_{k}\right), \lambda \gamma, \mu\right) \\
& =S_{\text {firm }}\left(W W^{T} \tilde{u}_{k}+\gamma W F^{T} U^{T}\left(y-U F W^{T} \tilde{u}_{k}\right), \lambda \gamma, \mu\right) \\
& =S_{\text {firm }}\left(W\left(W^{T} \tilde{u}_{k}+\gamma F^{T} U^{T}\left(y-U F W^{T} \tilde{u}_{k}\right)\right), \lambda \gamma, \mu\right) .
\end{aligned}
$$

By substituting the coefficients $\tilde{u}_{k}$ and $\tilde{u}_{k+1}$ with images $x_{k}=\Phi \tilde{u}_{k}$ and $x_{k+1}=\Phi \tilde{u}_{k+1}$, we get that

$$
x_{k+1}=W^{T} S_{f i r m}\left(W\left(x_{k}+\gamma F^{T} U^{T}\left(y-U F x_{k}\right)\right), \lambda \gamma, \mu\right) .
$$

In summary, the computation procedure for problem (7) is presented in the following algorithm.

\section{Algorithm 1}

Parameters: $\lambda, \gamma$ and $\mu$

Initialization: $x_{0}$

While not converge, do

$x_{k+1}=W^{T} S_{\text {firm }}\left(W\left(x_{k}+\gamma F^{T} U^{T}\left(y-U F x_{k}\right), \lambda \gamma, \mu\right)\right.$

Output: $x$

Furthermore, the same accelerating strategy as FISTA [28] is introduced resulting in the following algorithm.

\section{Algorithm 2}

Parameters: $\lambda, \gamma$ and $\mu$

Initialization: $t_{0}=1, x_{0}, \tilde{x}_{0}$

While not converge, do

$$
\begin{aligned}
& x_{k+1}=W^{T} S_{f i r m}\left(W\left(\tilde{x}_{k}+\gamma F^{T} U^{T}\left(y-U F \tilde{x}_{k}\right), \lambda \gamma, \mu\right)\right. \\
& t_{k+1}=\frac{1+\sqrt{1+4 t_{k}^{2}}}{2} \\
& \tilde{x}_{k+1}=x_{k+1}+\frac{t_{k}-1}{t_{k+1}}\left(x_{k+1}-x_{k}\right)
\end{aligned}
$$

Output: $x$ 


\section{Numerical experiments}

In this section, we conduct several numerical experiments to illustrate the performance of the proposed nonconvex model (4). We test five images shown in Figure 2, where Figure 2(c)-(b) are three T2-weighted brain images with slices 7,10 and 27 respectively, see in [10].

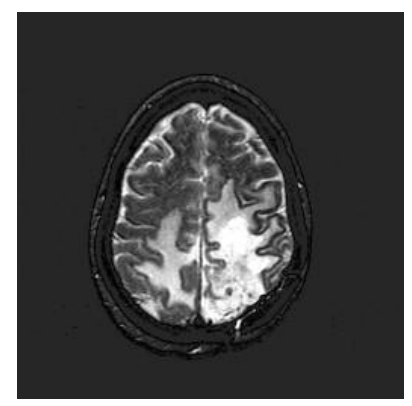

(a)

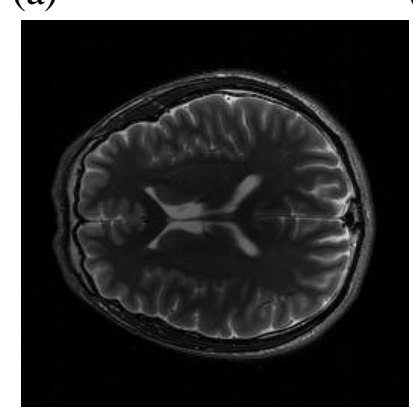

(d)

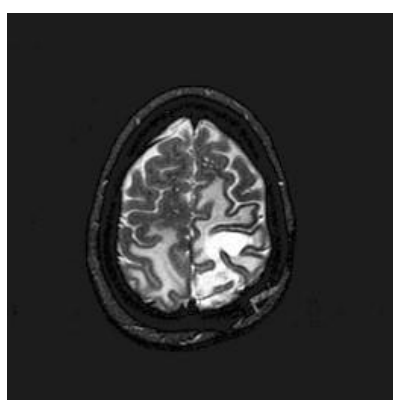

(b)

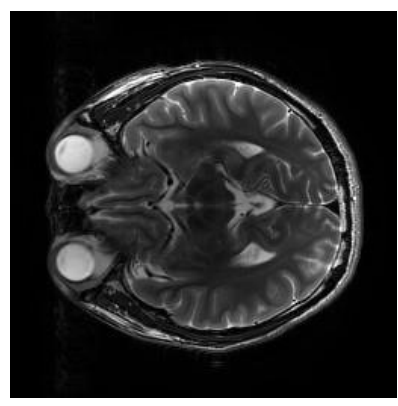

(c)

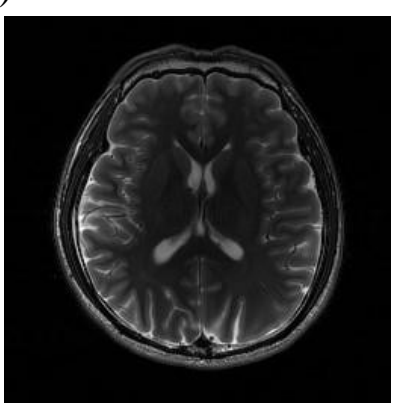

(e)

Figure 2: Standard images used in the experiments.

To test the performance of the proposed method with different sampling patterns, we use the Cartesian mask, pseudo-radial mask [10], and 30\% sampled 2D Gaussian mask [10] shown in Figure 3(a)-(c) respectively, to undersample the k-space data of the images in Figure 3.
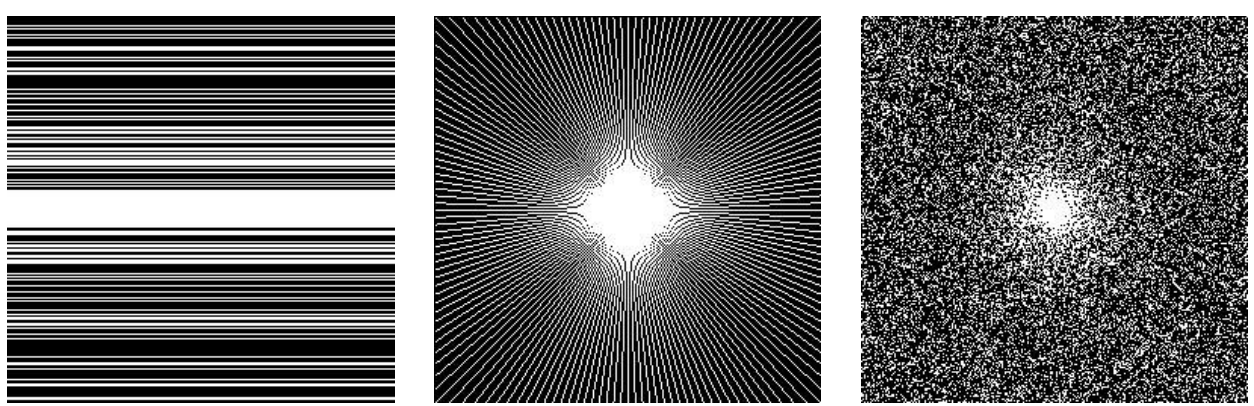

Figure 3: Standard images used in the experiments 
We use two tight frames: the Contourlet [30] and the shift-invariant discrete wavelet transform (SIDWT) [29], and the Daubechies wavelet with 4 decomposition levels is utilized for SIDWT in all experiments.

We compare our method with two methods SFISTA [32] and pFISTA [10],

which are two algorithms to solve the convex model (2). We implement all methods by using MATLAB 7.7 (R2008b), and run on an Intel Pentium Dual CPU at 2.60 $\mathrm{GHz}$ and 4GB of memory. The quality of the reconstructed images is measured by Peak-signal-to-noise ratio (PSNR), and the relative $l_{2}$ norm error (RLNE). They are defined as follows:

$$
\begin{gathered}
\mathrm{PSNR}=20 \log _{10} \frac{255}{\frac{1}{m n}\left\|u-f^{*}\right\|_{2}}, \\
\mathrm{RLNE}=\frac{\left\|u-f^{*}\right\|_{2}}{\left\|f^{*}\right\|_{2}} .
\end{gathered}
$$

Where $f^{*}$ is the fully sampled image, and $u$ is the reconstructed image. The stopping criterion of all the three methods is that the relative difference between the successive iterate of the reconstructed image satisfies the following inequality:

$$
\frac{\left\|u^{(i+1)}-u^{(i)}\right\|_{2}}{\left\|u^{(i+1)}\right\|_{2}}<10^{-5} .
$$

The parameters for all the methods are set as follows. For SFISTA, the step size $\gamma_{s}=1 /(1+1 / \mu)$ [11,31], and $\mu$ is adjusted as in [31]. For pFISTA, the step size $\gamma_{p}=1$ is set for both promising reconstruction performance and fast speed as in [10]. For our method, the step size $\gamma$ is also set to be 1 . For fair comparison, the regularization parameters, $\lambda_{s}$ of SFISTA, $\lambda_{p}$ of pFISTA and $\lambda$ of our method are set such that the optimal PSNR values are achieved.

\subsection{Numerical experiments without additive noise}

In the first numerical experiment, we undersample k-space data of the images by three masks shown in Figure 3 (a)-(c) (the Cartesian mask, the Pseudo-radial mask and 30\% sampled 2D Gaussian mask respectively), and SIDWT and the Contourlet are utilized to reconstruct MR images.

In Tables 1-3, we list the PSNR values and the RLNE values for SFISTA, pFISTA and our method for five images to show the effectiveness of the proposed method. We see that our method is the best in terms of PSNR values and RLNE values. Take 
the case of the images in Figure 2 (b) and (e) (undersampled by the masks in Figure 3 (a) and (b)), the reconstructed images by all the methods are shown in Figures 45 (c)-(e), and the difference images of the restored images in Figures 4-5 (f)-(h).

Table 1: PSNR(dB) values and RLNE for various methods for the test images undersampled by the Cartesian mask

\begin{tabular}{|c|c|c|c|c|c|c|}
\hline \multirow{2}{*}{ Image frame } & \multicolumn{3}{|c|}{ PSNR } & \multicolumn{3}{c|}{ RLNE } \\
\cline { 2 - 7 } & SFISTA & pFISTA & Proposed & SFISTA & pFISTA & Proposed \\
\hline Figure 2(a) SIDWT & 35.98 & 35.95 & 38.87 & 0.0983 & 0.0982 & 0.0577 \\
\hline Contourlet & 34.54 & 34.59 & 36.18 & 0.1158 & 0.1146 & 0.0891 \\
\hline Figure 2(b) SIDWT & 36.69 & 37.54 & 38.88 & 0.1055 & 0.1069 & 0.0787 \\
\hline Contourlet & 35.46 & 35.57 & 37.97 & 0.1241 & 0.1226 & 0.0891 \\
\hline Figure 2(c) SIDWT & 34.32 & 34.42 & 35.75 & 0.1379 & 0.1354 & 0.0858 \\
\hline Contourlet & 33.75 & 33.77 & 34.04 & 0.1488 & 0.1485 & 0.1157 \\
\hline Figure 2(d)SIDWT & 37.37 & 37.28 & 38.25 & 0.1333 & 0.1345 & 0.0769 \\
\hline Contourlet & 36.44 & 36.44 & 39.74 & 0.1475 & 0.1460 & 0.1056 \\
\hline Figure 2(e) SIDWT & 35.82 & 35.82 & 38.75 & 0.1225 & 0.1226 & 0.0781 \\
\hline Contourlet & 37.00 & 37.04 & 38.78 & 0.1347 & 0.1348 & 0.1047 \\
\hline
\end{tabular}

Table 2: PSNR(dB) values and RLNE for various methods for the test images undersampled by the pseudo-radial mask

\begin{tabular}{|c|r|c|c|c|c|c|}
\hline \multirow{2}{*}{ Image frame } & \multicolumn{3}{|c|}{ PSNR } & \multicolumn{3}{c|}{ RLNE } \\
\cline { 2 - 7 } & SFISTA & pFISTA & Proposed & SFISTA & pFISTA & Proposed \\
\hline Figure 2(a) SIDWT & 39.32 & 39.47 & 39.84 & 0.0433 & 0.0426 & 0.0408 \\
\hline Contourlet & 36.48 & 36.45 & 36.70 & 0.0601 & 0.0603 & 0.0586 \\
\hline Figure 2(b) SIDWT & 39.32 & 39.49 & 39.91 & 0.0433 & 0.0425 & 0.0405 \\
\hline Contourlet & 38.16 & 38.16 & 38.26 & 0.0579 & 0.0579 & 0.0573 \\
\hline Figure 2(c) SIDWT & 34.58 & 34.57 & 36.29 & 0.0965 & 0.0966 & 0.0833 \\
\hline Contourlet & 33.64 & 33.63 & 34.27 & 0.1168 & 0.1170 & 0.1097 \\
\hline Figure 2(d)SIDWT & 37.91 & 37.94 & 38.60 & 0.0904 & 0.0902 & 0.0708 \\
\hline Contourlet & 35.99 & 35.99 & 36.71 & 0.1112 & 0.1114 & 0.1036 \\
\hline Figure 2(e) SIDWT & 35.80 & 35.78 & 36.19 & 0.0959 & 0.0961 & 0.0835 \\
\hline Contourlet & 35.24 & 35.23 & 35.82 & 0.1121 & 0.1126 & 0.1059 \\
\hline
\end{tabular}


Table 3: PSNR(dB) values and RLNE for various methods for the test images undersampled by 30\% sampled 2D Gaussian mask

\begin{tabular}{|c|c|c|c|c|c|c|}
\hline \multirow{2}{*}{ Image frame } & \multicolumn{3}{|c|}{ PSNR } & \multicolumn{3}{c|}{ RLNE } \\
\cline { 2 - 7 } & SFISTA & pFISTA & Proposed & SFISTA & pFISTA & Proposed \\
\hline Figure 2(a) SIDWT & 40.21 & 40.35 & 42.97 & 0.0497 & 0.0494 & 0.0333 \\
\hline Contourlet & 33.67 & 33.77 & 36.03 & 0.0940 & 0.0931 & 0.0690 \\
\hline Figure 2(b) SIDWT & 43.02 & 43.51 & 43.65 & 0.0416 & 0.0397 & 0.0347 \\
\hline Contourlet & 35.30 & 38.16 & 37.62 & 0.0911 & 0.0897 & 0.0672 \\
\hline Figure 2(c) SIDWT & 33.07 & 32.94 & 37.71 & 0.1103 & 0.1122 & 0.0725 \\
\hline Contourlet & 31.90 & 32.04 & 32.43 & 0.1460 & 0.1443 & 0.1138 \\
\hline Figure 2(d)SIDWT & 40.12 & 40.11 & 42.06 & 0.0915 & 0.0922 & 0.0750 \\
\hline Contourlet & 34.59 & 34.79 & 36.66 & 0.1436 & 0.1411 & 0.1139 \\
\hline Figure 2(e) SIDWT & 38.52 & 38.87 & 40.91 & 0.0993 & 0.0958 & 0.0785 \\
\hline Contourlet & 34.11 & 34.29 & 36.36 & 0.1396 & 0.1374 & 0.1093 \\
\hline
\end{tabular}

The regularization parameters $\left(\lambda_{s}, \lambda_{p}, \lambda\right)$ are set to be $(0.001,0.001,0.006),(0.004$, $0.004,0.004),(0.005,0.005,0.006),(0.001,0.001,0.006),(0.001,0.001,0.004)$ for SFISTA, PFISTA and our method respectively, and the parameter $\mu$ in Algorithm 2 is $0.12,0.2,0.18,0.12,0.08$, when the images in Figure 2 (a)-(e) are undersampled by the Cartesian mask and reconstructed by SIDWT.

The regularization parameters $\left(\lambda_{s}, \lambda_{p}, \lambda\right)$ are set to be $(0.0004,0.0004,0.0004)$, (0.0004, 0.0004, 0.0004), (0.0006, 0.0006, 0.0006), (0.0004, 0.0004, 0.0004), $(0.0004,0.0004,0.0004)$ for SFISTA, PFISTA and our method respectively, and the parameter $\mu$ in Algorithm 2 is 0.2, 0.2, 0.18, 0.2, 0.2, when the images in Figure 2 (a)-(e) are undersampled by the Cartesian mask and reconstructed by Contourlet. The regularization parameters $\left(\lambda_{s}, \lambda_{p}, \lambda\right)$ are set to be $(0.0004,0.0004,0.0006)$, (0.0002, 0.0002, 0.0002), (0.0004, 0.0004, 0.0006), (0.0003, 0.0003, 0.0006), $(0.0004,0.0004,0.0006)$ for SFISTA, PFISTA and our method respectively, and the parameter $\mu$ in Algorithm 2 is $0.12,0.5,0.3,0.12,0.3$, when the images in Figure 2 (a)-(e) are undersampled by the pseudo-radial mask and reconstructed by SIDWT.

The regularization parameters $\left(\lambda_{s}, \lambda_{p}, \lambda\right)$ are set to be $(0.0006,0.0006,0.0006)$, (0.0008, 0.0008, 0.004), (0.0006, 0.0006, 0.0006), (0.0006, 0.0006, 0.0006), $(0.0004,0.0004,0.0004)$ for SFISTA, PFISTA and our method respectively, and the parameter $\mu$ in Algorithm 2 is $0.3,0.36,0.48,0.3,0.4$, when the images in Figure 2 (a)-(e) are undersampled by the pseudo-radial mask and reconstructed by Contourlet.

The regularization parameters $\left(\lambda_{s}, \lambda_{p}, \lambda\right)$ are set to be $(0.001,0.001,0.003)$, $(0.001,0.001,0.004),(0.003,0.003,0.003),(0.0004,0.0004,0.0008),(0.0006$, $0.0006,0.0006)$ for SFISTA, PFISTA and our method respectively, and the 
parameter $\mu$ in Algorithm 2 is 0.12, 0.16, 0.18, 0.72, 0.54, when the images in Figure 2 (a)-(e) are undersampled by the $30 \%$ sampled 2D Gaussian mask and reconstructed by SIDWT.

The regularization parameters $\left(\lambda_{s}, \lambda_{p}, \lambda\right)$ are set to be $(0.006,0.006,0.006)$, (0.006, 0.006, 0.006), (0.006, 0.006, 0.006), (0.006, 0.006, 0.006), (0.006, 0.006, 0.006) for SFISTA, PFISTA and our method, and the parameter $\mu$ in Algorithm 2 is $0.18,0.18,0.18,0.36,0.18$, when the images in Figure 2 (a)-(e) are undersampled by the $30 \%$ sampled 2D Gaussian mask and reconstructed by Contourlet.

\subsection{Numerical experiments with additive noise}

To demonstrate the ability of the proposed method in handling noise, Gaussian white noise with standard deviation 0.015 was added to both the real and the imaginary parts of the original k-space data.

In Tables 4-6, we list the PSNR values and the RLNE values for SFISTA, pFISTA and our method for five images to show the effectiveness of the proposed method in handling noise. We see that our method is the best in terms of PSNR values and RLNE values. Take the case of SIDWT, we give the restored images of all the methods in Figures 6-7.

Table 4: PSNR(dB) values and RLNE for various methods for the test images undersampled by the Cartesian mask and with Gaussian noise

\begin{tabular}{|c|c|c|c|c|c|c|}
\hline \multirow{2}{*}{ Image frame } & \multicolumn{3}{|c|}{ PSNR } & \multicolumn{3}{c|}{ RLNE } \\
\cline { 2 - 7 } & SFISTA & pFISTA & Proposed & SFISTA & pFISTA & Proposed \\
\hline Figure 2(a) SIDWT & 4.96 & 35.95 & 35.91 & 0.1101 & 0.1107 & 0.0833 \\
\hline Contourlet & 32.66 & 32.66 & 33.94 & 0.1240 & 0.1243 & 0.1040 \\
\hline Figure 2(b) SIDWT & 35.32 & 35.08 & 38.28 & 0.1160 & 0.1185 & 0.0809 \\
\hline Contourlet & 33.43 & 33.44 & 35.16 & 0.1339 & 0.1341 & 0.1067 \\
\hline Figure 2(c) SIDWT & 33.12 & 33.06 & 34.74 & 0.1496 & 0.1507 & 0.1013 \\
\hline Contourlet & 31.86 & 31.93 & 32.92 & 0.1620 & 0.1611 & 0.1307 \\
\hline Figure 2(d)SIDWT & 35.26 & 35.17 & 36.80 & 0.1597 & 0.1612 & 0.1376 \\
\hline Contourlet & 33.95 & 34.02 & 35.33 & 0.1691 & 0.1687 & 0.1462 \\
\hline Figure 2(e) SIDWT & 34.24 & 34.24 & 35.91 & 0.1459 & 0.1459 & 0.1219 \\
\hline Contourlet & 35.43 & 35.47 & 37.11 & 0.1518 & 0.1520 & 0.1294 \\
\hline
\end{tabular}


Table 5: PSNR(dB) values and RLNE for various methods for the test images undersampled by the pseudo-radial mask and with Gaussian noise

\begin{tabular}{|c|c|c|c|c|c|c|}
\hline \multirow{2}{*}{ Image frame } & \multicolumn{3}{|c|}{ PSNR } & \multicolumn{3}{c|}{ RLNE } \\
\cline { 2 - 7 } & SFISTA & pFISTA & Proposed & SFISTA & pFISTA & Proposed \\
\hline Figure 2(a) SIDWT & 37.26 & 36.96 & 37.77 & 0.0601 & 0.0619 & 0.0579 \\
\hline Contourlet & 35.55 & 35.50 & 36.03 & 0.0731 & 0.0738 & 0.0708 \\
\hline Figure 2(b) SIDWT & 38.64 & 38.38 & 39.06 & 0.0614 & 0.0629 & 0.0598 \\
\hline Contourlet & 36.90 & 36.84 & 37.25 & 0.0749 & 0.0759 & 0.0738 \\
\hline Figure 2(c) SIDWT & 31.27 & 30.99 & 30.87 & 0.1132 & 0.1163 & 0.1077 \\
\hline Contourlet & 30.25 & 30.22 & 30.87 & 0.1275 & 0.1281 & 0.1212 \\
\hline Figure 2(d)SIDWT & 36.14 & 35.91 & 36.91 & 0.1167 & 0.1192 & 0.1036 \\
\hline Contourlet & 35.08 & 35.04 & 35.84 & 0.1318 & 0.1329 & 0.1253 \\
\hline Figure 2(e) SIDWT & 33.22 & 33.00 & 34.34 & 0.1201 & 0.1226 & 0.1094 \\
\hline Contourlet & 34.43 & 34.41 & 35.04 & 0.1306 & 0.1314 & 0.1254 \\
\hline
\end{tabular}

Table 6: PSNR(dB) values and RLNE for various methods for the test images undersampled by $30 \%$ sampled $2 \mathrm{D}$ Gaussian mask and with Gaussian noise

\begin{tabular}{|c|c|c|c|c|c|c|}
\hline \multirow{2}{*}{ Image frame } & \multicolumn{3}{|c|}{ PSNR } & \multicolumn{3}{c|}{ RLNE } \\
\cline { 2 - 7 } & SFISTA & pFISTA & Proposed & SFISTA & pFISTA & Proposed \\
\hline Figure 2(a) SIDWT & 36.32 & 35.99 & 37.87 & 0.0745 & 0.0768 & 0.0580 \\
\hline Contourlet & 34.13 & 34.19 & 35.85 & 0.0987 & 0.0985 & 0.0812 \\
\hline Figure 2(b) SIDWT & 38.05 & 37.68 & 40.40 & 0.0718 & 0.0742 & 0.0589 \\
\hline Contourlet & 35.70 & 35.77 & 37.53 & 0.0971 & 0.0970 & 0.0811 \\
\hline Figure 2(c) SIDWT & 31.62 & 31.42 & 35.93 & 0.1276 & 0.1303 & 0.0936 \\
\hline Contourlet & 32.22 & 32.29 & 32.93 & 0.1518 & 0.1512 & 0.1300 \\
\hline Figure 2(d)SIDWT & 36.32 & 36.12 & 39.42 & 0.1276 & 0.1303 & 0.0991 \\
\hline Contourlet & 34.98 & 35.06 & 36.83 & 0.1529 & 0.1527 & 0.1302 \\
\hline Figure 2(e) SIDWT & 35.42 & 35.26 & 37.00 & 0.1302 & 0.1323 & 0.0982 \\
\hline Contourlet & 34.50 & 34.58 & 35.97 & 0.1484 & 0.1479 & 0.1292 \\
\hline
\end{tabular}

Take the case of the images in Figure 2 (b) and (d) (undersample by the masks in Figure 3 (b) and (c)), the restored images of by all the methods are shown in Figure 4-5(c)-(e), and the difference images of the restored images in Figure 4-5(f)-(h). The regularization parameters $\left(\lambda_{s}, \lambda_{p}, \lambda\right)$ are set to be $(0.002,0.002,0.008)$, (0.008, 0.008, 0.008), (0.004, 0.004, 0.004), (0.005, 0.005, 0.005), (0.003, 0.003, 0.003 ) for SFISTA, PFISTA and our method respectively, and the parameter $\mu$ in Algorithm 2 is $0.4,0.24,0.12,1,0.6$, when the images in Figure 2(a)-(e) are undersampled by the Cartesian mask and reconstructed by SIDWT.

The regularization parameters $\left(\lambda_{s}, \lambda_{p}, \lambda\right)$ are set to be 0.007 for SFISTA, PFISTA and our method respectively, and the parameter $\mu$ in Algorithm 2 is 0.35, 0.35, 
0.35, 0.56, 0.15, when the images in Figure 2(a)-(e) are undersampled by the Cartesian mask, added the Gaussian noise with standard deviation 0.01, and reconstructed by Contourlet.

The regularization parameters $\left(\lambda_{s}, \lambda_{p}, \lambda\right)$ are set to be $(0.006,0.006,0.006)$, (0.006, 0.006, 0.006), (0.008, 0.008, 0.008), (0.006, 0.006, 0.006), (0.006, 0.006, $0.006)$ for SFISTA, PFISTA and our method respectively, and the parameter $\mu$ in Algorithm 2 is $0.36,0.36,0.24,0.18,0.36$, when the images in Figure 2(a)-(e) are undersampled by the pseudo-radial mask and reconstructed by SIDWT.

The regularization parameters $\left(\lambda_{s}, \lambda_{p}, \lambda\right)$ are set to be 0.003 for SFISTA, PFISTA and our method respectively, and the parameter $\mu$ in Algorithm 2 is 0.45, 0.45, 4.5, 3, 3, when the images in Figure 2(a)-(e) are undersampled by the pseudo-radial mask, added the Gaussian noise with standard deviation 0.01, and reconstructed by Contourlet.

The regularization parameters $\left(\lambda_{s}, \lambda_{p}, \lambda\right)$ are set to be $(0.006,0.006,0.006),(0.006$, 0.006, 0.006), (0.006, 0.006, 0.006), (0.006, 0.006, 0.006), $(0.006,0.006,0.006)$ for SFISTA, PFISTA and our method respectively, and the parameter $\mu$ in Algorithm 2 is 0.18 , when the images in Figure 2(a)-(e) are undersampled by the $30 \%$ sampled 2D Gaussian mask and reconstructed by SIDWT.

The regularization parameters $\left(\lambda_{s}, \lambda_{p}, \lambda\right)$ are set to be $(0.003,0.003,0.003),(0.003$, 0.003, 0.003), (0.003, 0.003, 0.003), (0.003, 0.003, 0.003), $(0.003,0.003,0.003)$ for SFISTA, PFISTA and our method respectively, and the parameter $\mu$ in Algorithm 2 is $3,3,3,0.15,3$, when the images in Figure 2(a)-(e) are undersampled by the $30 \%$ sampled 2D Gaussian mask, added the Gaussian noise with standard deviation 0.01, and reconstructed by Contourlet. 


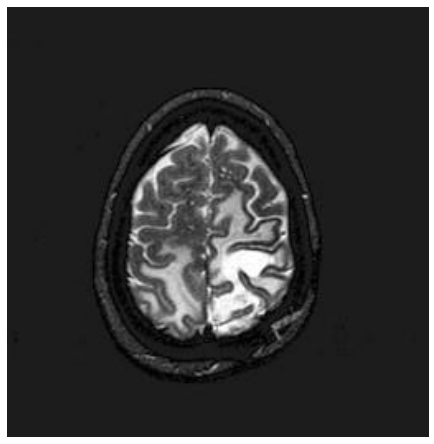

(a)

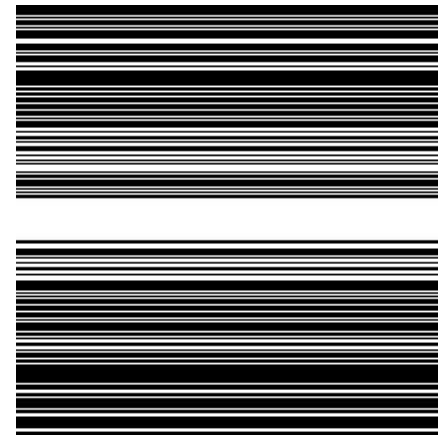

(b)

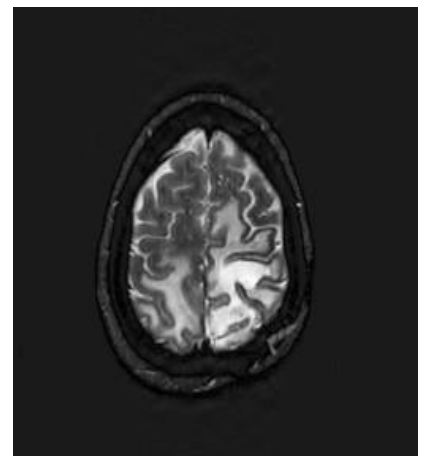

(c)

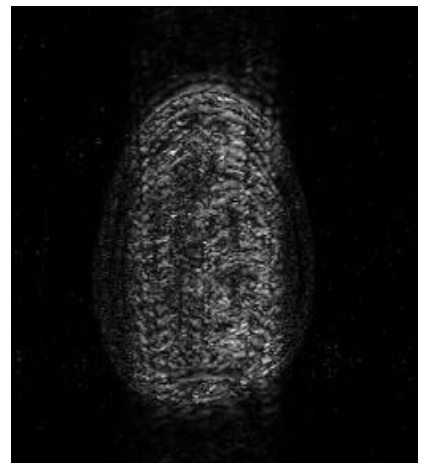

(e)

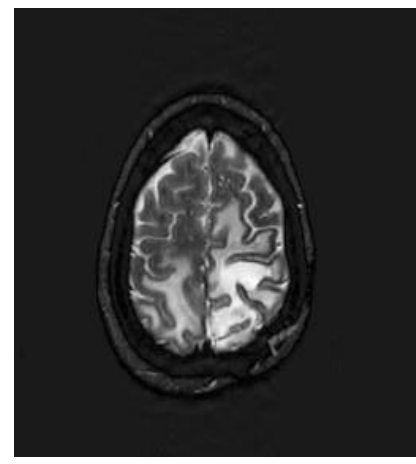

(d)

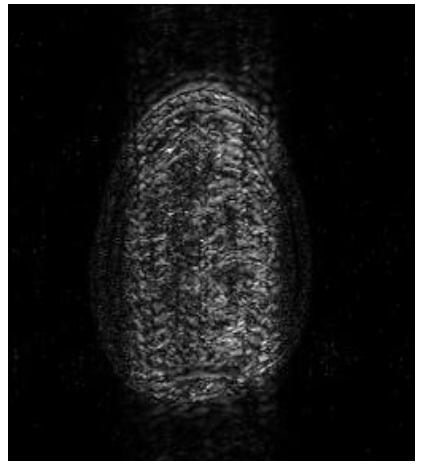

(f)

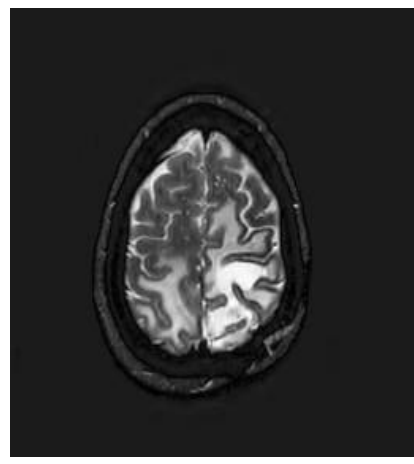

(e)

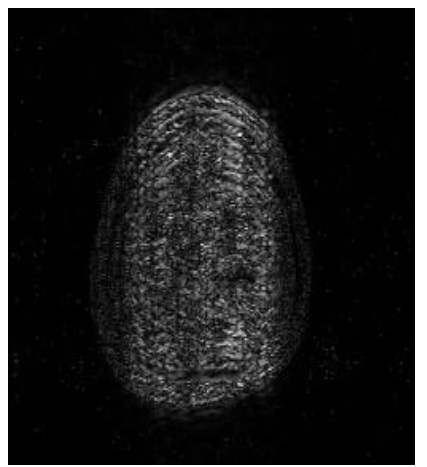

(h)

Figure 4: Reconstructed images of different methods using SIDWT on the image in Figure 2 (a) which is undersampled by the Cartesian mask. (a) is the original image. (b) is the Cartesian mask. (c)-(e) are the reconstructed images of (a). (f)-(h) are the different images of (c)-(e) to the ground truth image 


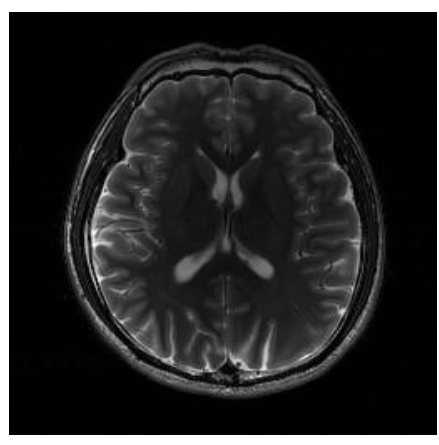

(a)

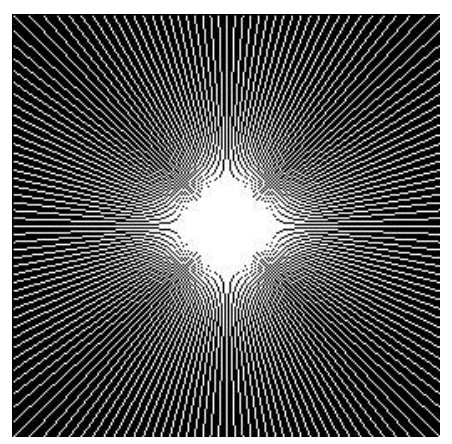

(b)

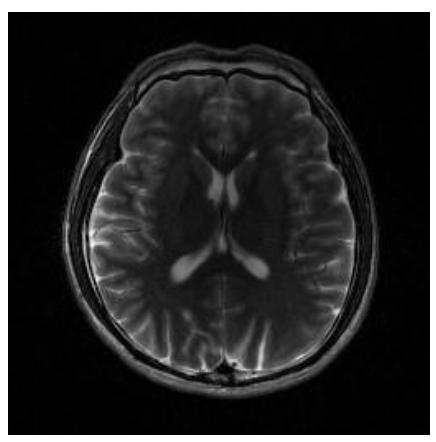

(c)

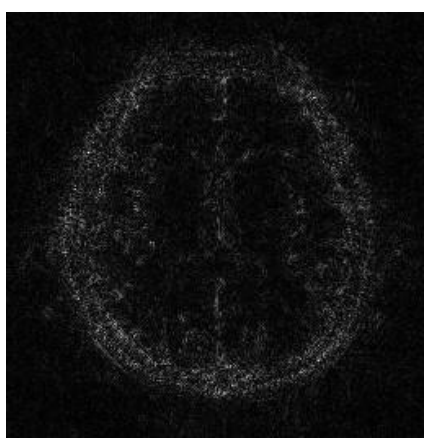

(f)

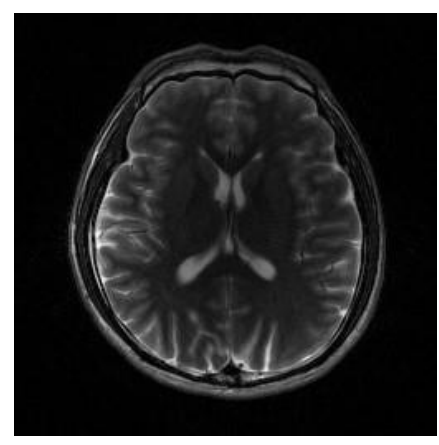

(d)

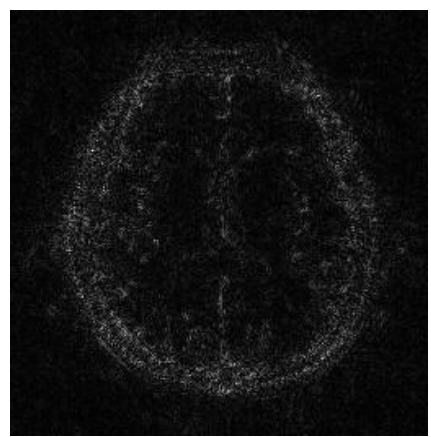

(g)

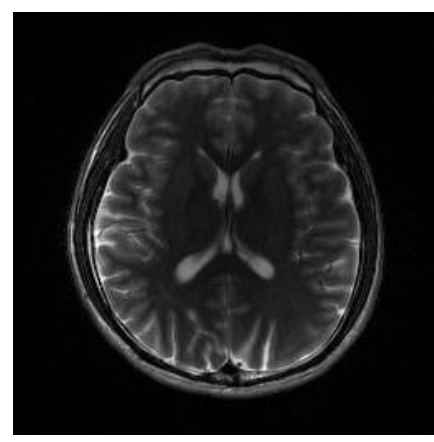

(e)

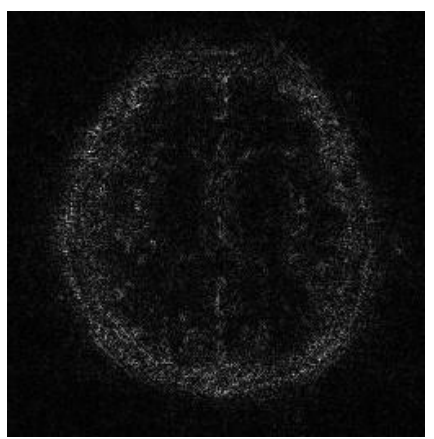

(h)

Figure 5: Reconstructed images of different methods using Contourlet on the image in Figure 2 (a) which is undersampled by the pseudo-radial mask. (a) is the original image. (b) is the pseudo-radial mask. (c)-(e) are the reconstructed images of (a). (f)-(h) are the different images of (c)-(e) to the ground truth image. 


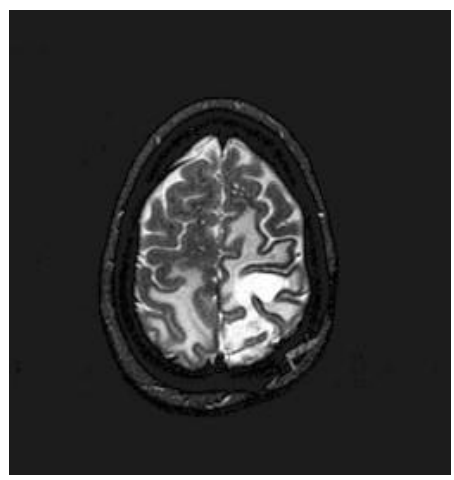

(a)

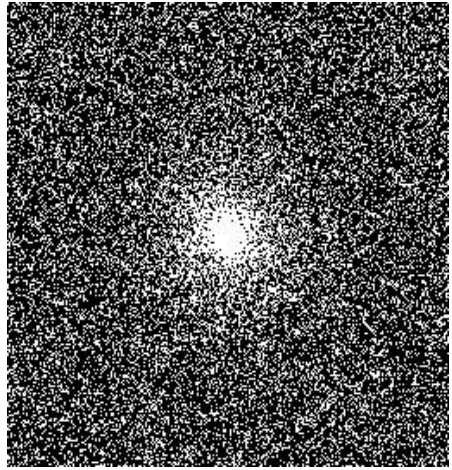

(b)

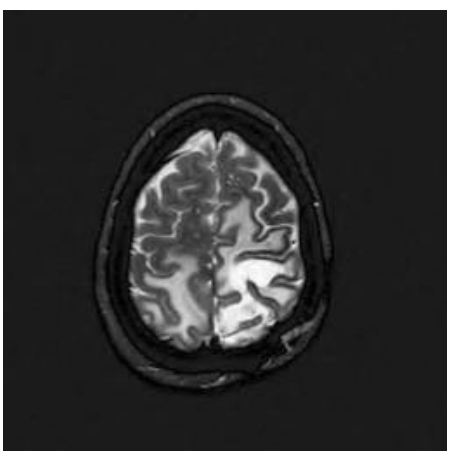

(c)

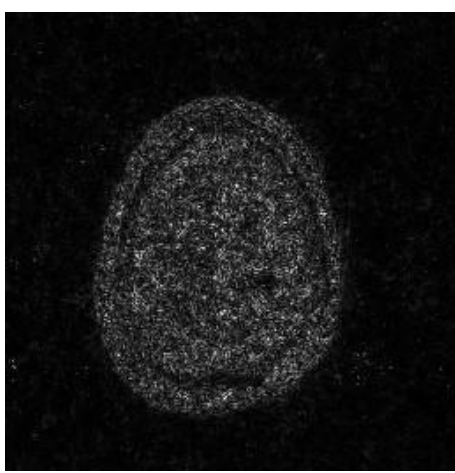

(f)

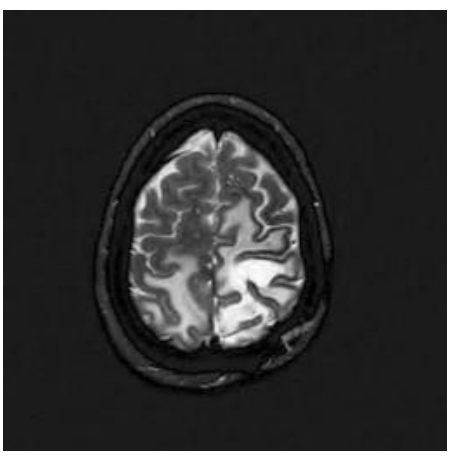

(d)

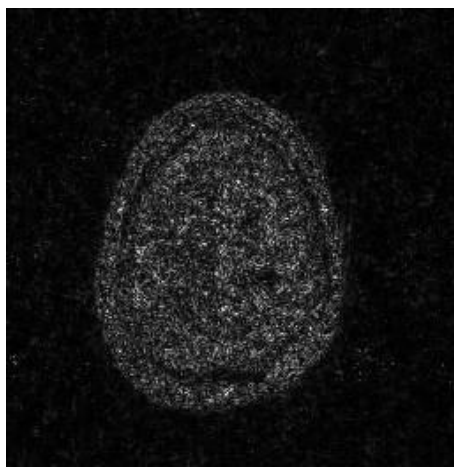

(g)

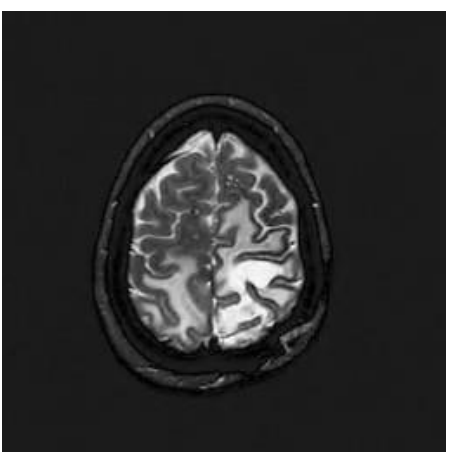

(e)

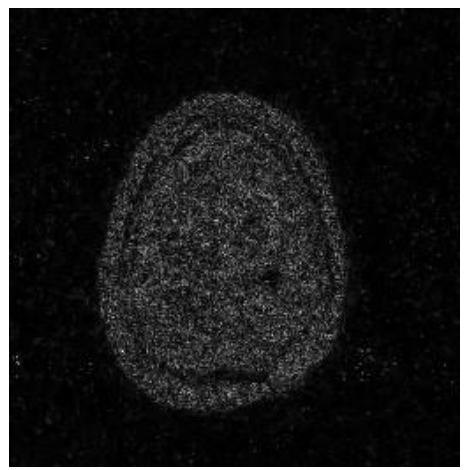

(h)

Figure 6: Reconstructed images of different methods using SIDWT on the image in Figure 2 (a) which is undersampled by the 30\% sampled 2D Gaussian mask and with Gaussian noise. (a) is the original image. (b) is the 30\% sampled 2D Gaussian mask. (c)-(e) are the reconstructed images of (a). (f)-(h) are the different images of (c)-(e) to the ground truth image. 


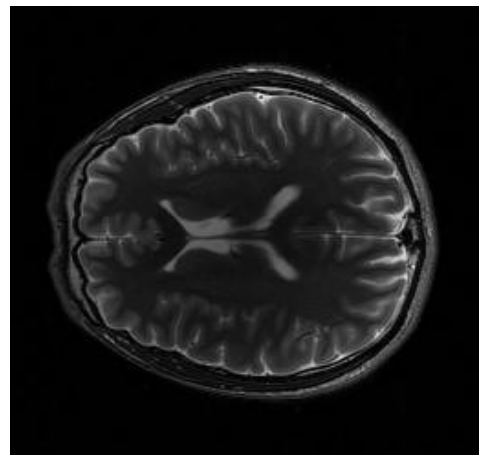

(a)

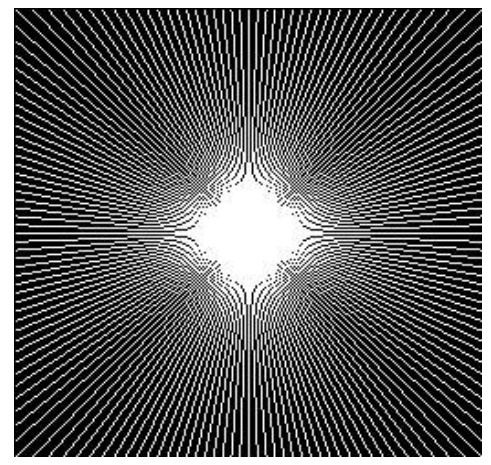

(b)

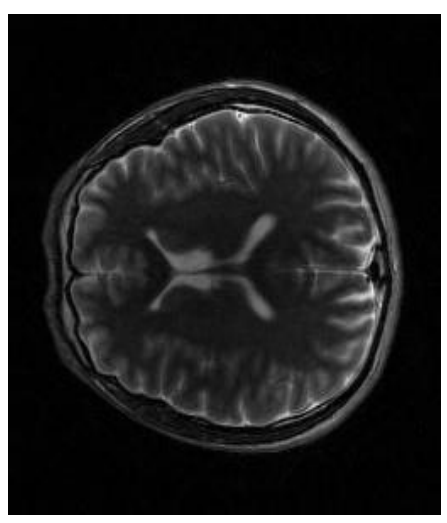

(c)

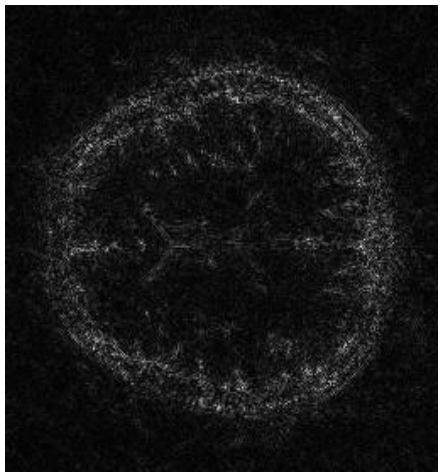

(f)

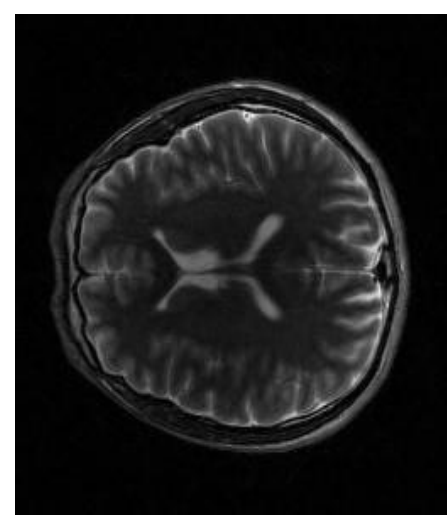

(d)

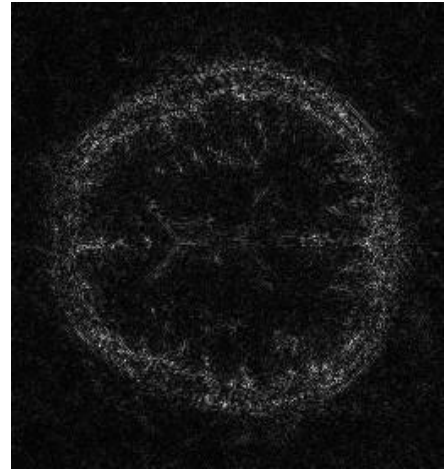

(g)

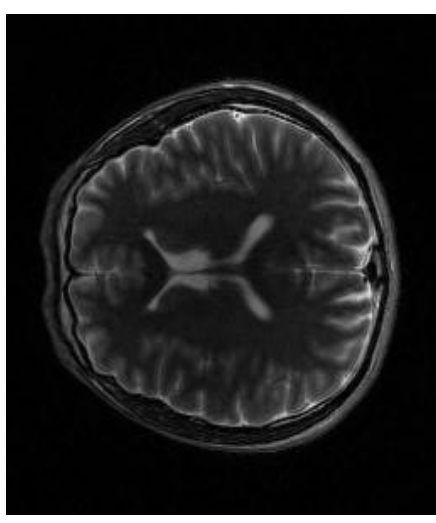

(e)

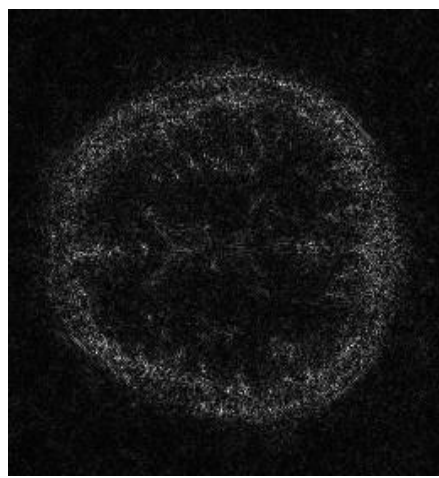

(h)

Figure 7: Reconstructed images of different methods using contourlet on the image in Figure 2 (a) which is undersampled by the pseudo-radial mask and with Gaussian noise. (a) is the original image. (b) is the pseudo-radial mask. (c)-(e) are the reconstructed images of (a). (f)-(h) are the different images of (c)-(e) to the ground truth image. 


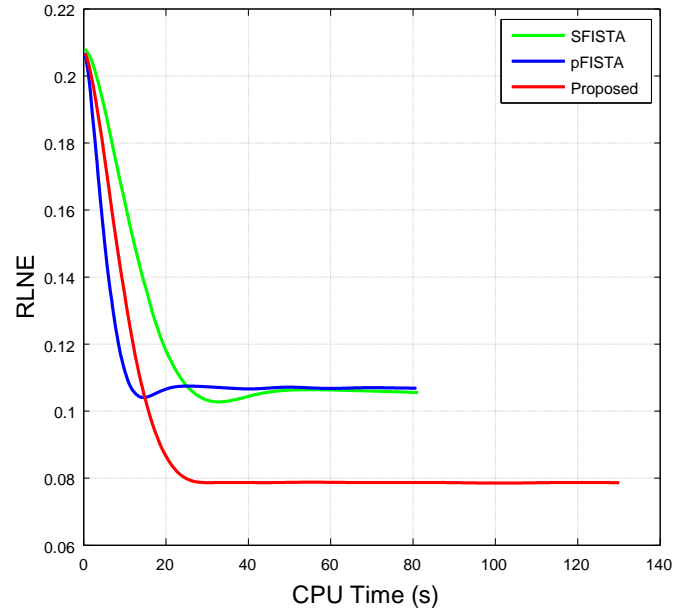

(a)

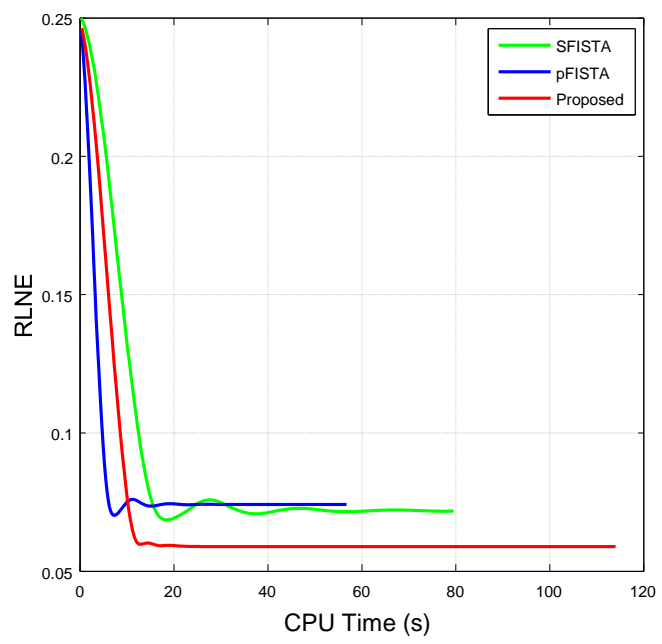

(c)

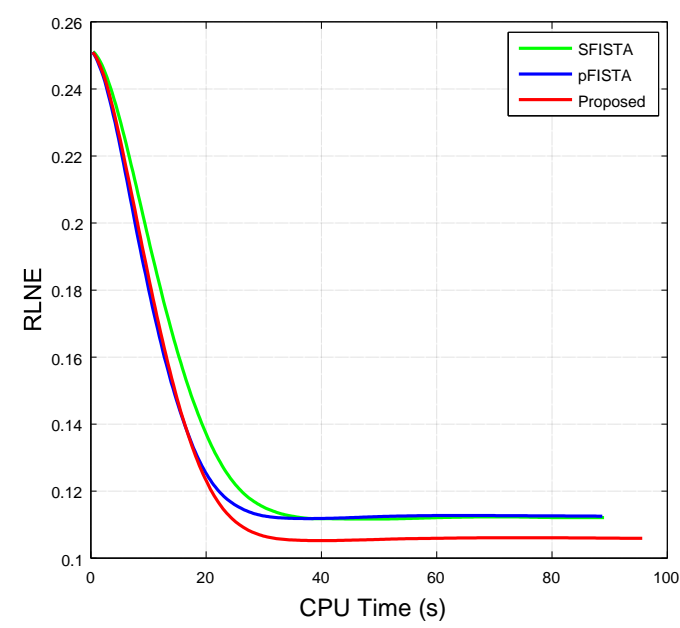

(b)

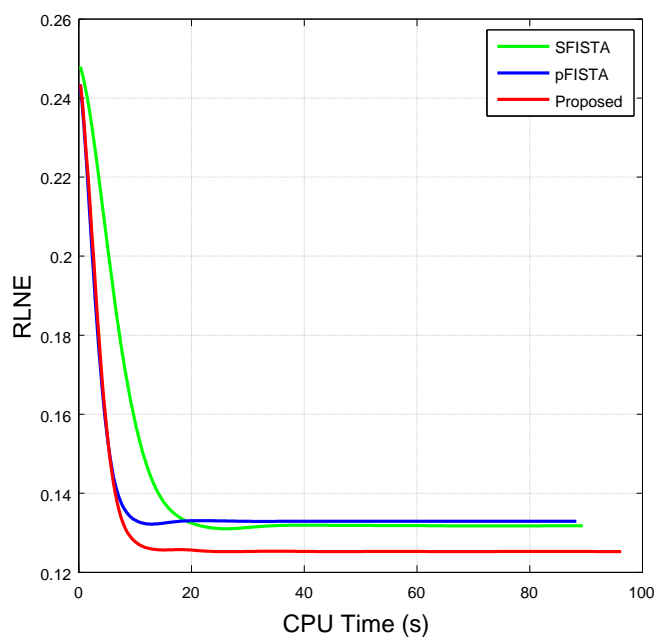

(d)

Figure 8: (a) and (b) are the empirical convergence results without noise and (c)-(d) with noise. The brain image in Figure 2(b), the Cartesian mask and SIDWT are adopted in (a). The brain image in Figure 2 (e), the pseudo-radial mask and contourlet are adopted in (b). The brain image in Figure 2 (b), the $30 \%$ sampled 2D Gaussian mask and SIDWT are adopted in (c). The brain image in Figure 2 (d), the pseudo-radial mask and contourlet are adopted in 


\section{Conclusion}

In this paper, we propose a variation model for compressed sensing recovery. This model takes advantage of the wavelet tight frame and nonconvex shrinkage penalties. We discuss the condition of strict convexity of the model. Various numerical results were reported to exhibit the performance of the new model in terms of the convergence speed and reconstruction errors.

\section{ACKNOWLEDGEMENTS}

The work is supported by the Open Project of Jiangsu Key Laboratory of Numerical Simulation of Large Scale Complex Systems under Grant No. 201803, Qing Lan Project of Education Department of Jiangsu Province, and the Natural Science Foundation of Jiangsu Province of China under grant BK20200267.

\section{References}

[1] Figueiredo, Mario A. T. and Bioucas-Dias, José M. (2010). Restoration of poissonian images using alternating direction optimization, IEEE transactions on image processing: a publication of the IEEE Signal Processing Society, 19(12), 3133-3145.

[2] Elad, M., Starck, J. L., Querre, P. and Donoho, D. L. (2005). Simultaneous cartoon and texture image inpainting using morphological component analysis (MCA), Applied and Computational Harmonic Analysis, 19, 340-358.

[3] Cai, J. F., Osher, S. and Shen, Z. (2010). Split bregman methods and frame based image restoration, Multiscale Modeling and Simulation, 8(2), 337-369.

[4] Cai, J. F., Osher, S. and Shen, Z. (2009). Linearized Bregman iterations for frame-based image deblurring, SIAM J. Imaging Sci., 2,226-252.

[5] Daubechies, I., Teschke, G. and Vese, L. (2007). Iteratively solving linear inverse problems under general convex constraints, Inverse Problem Imaging, 1, 29-46.

[6] Elad, M., Milanfar, P. and Rubinstein, R. (2007). Analysis versus synthesis in signal priors, Inverse problems, 23(3), 947.

[7] Candes, E. J., Eldar, Y. C., Needell, D. and Randall, P. (2011). Compressed sensing with coherent and redundant dictionaries, Applied and Computational Harmonic Analysis, 31(1), 59-73.

[8] Nam, S., Davies, M. E., Elad, M. and Gribonval, R. (2013). The cosparse analysis model and algorithms, Applied and Computational Harmonic Analysis, 34(1),30-56.

[9] Cai, J. F., Osher, S. and Shen, Z. (2009). Split Bregman methods and frame based image restoration, Multiscale modeling and simulation, 8(2),337-369.

[10] Liu, Y., Zhan, Z., Cai, J. F., Di, G., Zhong, C. and Qu, X. (2016). Projected iterative soft-thresholding algorithm for tight frames in compressed sensing magnetic resonance imaging, IEEE Transactions on Medical Imaging, 35(9), 2130-2140. 
[11] Beck, A. and Teboulle, M. (2009). A fast iterative shrinkage-thresholding algorithm for linear inverse problems, SIAM J. Imaging Sciences, 2(1), 183202.

[12] Nikolova, M. (2000). Local strong homogeneity of a regularized estimator, SIAM Journal on Applied Mathematics.

[13] Nikolova, M. (2011). Energy minimization methods, Springer.

[14] Nikolova, M., Ng, M. K. and Tam, C.P. (2010). Fast nonconvex nonsmooth minimization methods for image restoration and reconstruction, IEEE Trans. Image Process., 19(12),3073-3088.

[15] Ding, Y. and Selesnick, I. W. (2015), Artifact-free Wavelet Denoising: Nonconvex Sparse Regularization, Convex Optimization, IEEE Signal Processing Letters, 22(9),1364-1368.

[16] Woodworth, J., Chartrand, R. (2016). Compressed sensing recovery via nonconvex shrinkage penalties, Inverse Problems, 32(7),75004-75028.

[17] Candes, E. J., Romberg, J. and Tao, T. (2006). Robust uncertainty principles: exact signal reconstruction from highly incomplete frequency information, IEEE Transactions on Information Theory, 52(2),489-509.

[18] Donoho, D. L. (2006). Compressed sensing, IEEE Transactions on Information Theory, 52(4),1289-1306.

[19] Bruckstein, A. M., Donoho, D. L. and Elad, M. (2009). From sparse solutions of systems of equations to sparse modeling of signals and images, SIAM Review,51(1),34-81.

[20] Cai, T. T. and Zhang, A. (2013). Sharp rip Bound for sparse signal and lowrank matrix recovery, Applied and Computational Harmonic Analysis, 35(1):74-93.

[21] Davies, M. E. and Gribonval, R. (2009). Restricted isometry constants where $l^{p}$ sparse recovery can fail for $0<p \leq 1$, IEEE Transactions on Information Theory,55,2203-2214.

[22] Elad, M. and Bruckstein, A. M. (2002). A generalized uncertainty princple and sparse representation in pairs of bases, IEEE Transactions on Information Theory, 48(9),2558-2567.

[23] Chartrand, R. (2009). Fast algorithms for nonconvex compressive sensing: MRI reconstruction from very few data, IEEE International Symposium on Biomedical Imaging.

[24] Chartrand, R. (2014). Shrinkage mappings and their induced penalty functions, IEEE International Conference on Acoustics, Speech and Signal Processing.

[25] Chartrand, R., Sidky, E. Y. and Pan, X. (2013). Nonconvex compressive sensing for X-ray CT: an algorithm comparison, Asilomar Conference on Signals, Systems and Computers.

[26] Hiriart-Urruty, J. B. and Lemarechal, C. (2001). Fundamentals of convex analysis. Springer Science and Business Media, 2001. 
[27] Daubechies, I., Defrise, M. and Mol, C.D. (2004). An iterative thresholding algorithm for linear inverse problems with a sparsity constraint. Communications on pure and applied mathematics, 57(11),1413-1457.

[28] Beck, A. and Teboulle, M. (2009). A Fast Iterative Shrinkage-Thresholding Algorithm for Linear Inverse Problems. Siam J Imaging Sciences, 2(1),183202.

[29] Baker, C. A., King, K. F., Liang, D. and Yang, L. (2011). Translationalinvariant dictionaries for compressed sensing in magnetic resonance imaging. Proceedings of the 8th IEEE International Symposium on Biomedical Imaging: From Nano to Macro.

[30] Do, M. N. and Vetterli, M. (2005). The contourlet transform: an efficient directional multiresolution image representation, IEEE Transactions on Image Processing ,14(12),2091-2106.

[31] Tan, Z., Eldar, Y. C., Beck, A. and Nehorai, A. (2014). Smoothing and decomposition for analysis sparse recovery, IEEE Transactions on Image Processing, 62(7),1762-1774. 